\title{
Geometric analysis of optical frequency conversion and its control in quadratic nonlinear media
}

\begin{abstract}
G. G. Luther
The Basic Research Institute in the Mathematical Sciences, Hewlett-Packard Laboratories, Filton Road, Stoke Gifford, Bristol BS12 6QZ, UK; Control and Dynamical Systems 107-81, Caltech, Pasadena, California 91125; Department of Mathematics, University of Notre Dame, Notre Dame, Indiana 46556; Department of Engineering Sciences and Applied Mathematics, McCormick School of Engineering and Applied Science, Northwestern University, 2145 Sheridan Road, Evanston, Illinois 60208
\end{abstract}

M. S. Alber

Department of Mathematics, University of Notre Dame, Notre Dame, Indiana 46556

J. E. Marsden

Control and Dynamical Systems 107-81, Caltech, Pasadena, California 91125

\section{J. M. Robbins}

The Basic Research Institute in the Mathematical Sciences, Hewlett-Packard Laboratories, Filton Road, Stoke Gifford, Bristol BS12 6QZ, UK; School of Mathematics, University Walk, Bristol BS8 1TW, UK

Received June 1, 1999; revised manuscript received February 2, 2000

\begin{abstract}
We analyze frequency conversion and its control among three light waves using a geometric approach that enables the dynamics of the waves to be visualized on a closed surface in three dimensions. It extends the analysis based on the undepleted-pump linearization and provides a simple way to understand the fully nonlinear dynamics. The Poincaré sphere has been used in the same way to visualize polarization dynamics. A geometric understanding of control strategies that enhance energy transfer among interacting waves is introduced, and the quasi-phase-matching strategy that uses microstructured quadratic materials is illustrated in this setting for both type I and II second-harmonic generation and for parametric three-wave interactions. (C) 2000 Optical Society of America [S0740-3224(00)02006-3]
\end{abstract}

OCIS codes: $0190.0190,190.2640,000.3860,190.4410$

\section{INTRODUCTION}

Energy conversion among light waves occurs in optical materials where the interaction of the light with the material is well characterized by expansion of the polarizability up to at least second order in the electric field. Over a fixed length the conversion efficiency depends on a resonance condition among the frequencies and one among the wave numbers. Conversion efficiency also depends on the coupling strength among the waves. The magnitude of this coupling is given by the quadratic coefficient, $\chi^{(2)}$, in the amplitude expansion of the polarizability. ${ }^{1-3}$ The dynamical evolution of three waves resonantly coupled in this way is captured by the three-wave equations for their slowly varying envelopes. Because optical materials tend to have a relatively weak nonlinear response, there has been great interest in improving the effective response by microstructuring them. Here we view this as a nonlinear control problem and introduce a simple geometric picture for the dynamics and control of resonant-wave interactions.

Solutions of the three-wave equations have been known in nonlinear optics almost since its inception in the early 1960's. 4 Since this early work, an extensive literature on the analysis and application of these solutions in nonlinear optics has developed. In previous investigations the dynamics of the three-wave system was reduced with the aid of the Manley-Rowe relations to a single nonlinear oscillator $^{4-8}$ Features of the canonical Hamiltonian structure of the three-wave system have also been exploited. $^{6-8}$ In what follows we introduce a different approach.

The present approach is based on a systematic theory of Hamiltonian systems with symmetry, ${ }^{9}$ called reduction. For the three-wave equations the symmetries are just the phase invariances associated with the ManleyRowe relations, and the reduction procedure leads to a two-dimensional reduced phase space, the three-wave surface, in which the three wave amplitudes and their relative phase are incorporated simultaneously. In contrast, the standard approach reduces the three-wave system to a phase space for the modulus of a single wave amplitude. 
The three-wave surfaces are deformed spheres, and the dynamics on them is described by a simple geometrical construction. ${ }^{10,11}$ This construction provides a means of visualizing the dynamics of three-wave interactions, analogous to the use of the Poincare sphere for polarization dynamics, ${ }^{12-14}$ and it enables existing analysis to be readily comprehended in geometrical terms. In this way, recent techniques of geometric mechanics provide fresh insight into the dynamics of second-harmonic generation and parametric frequency conversion in quadratic, or $\chi^{(2)}$, media. In particular, this geometrical treatment facilitates the analysis of the control of three-wave interactions.

As early as 1962, Armstrong, Bloembergen, Ducuing, and Pershan ${ }^{4}$ proposed that the flow of energy among interacting light waves could be controlled by modulation of the sign of the susceptibility. In recent years these basic ideas have been implemented to simplify frequency conversion $^{15}$ and to improve optical parametric oscillators. $^{16}$ By formulating the dynamics in terms of the three-wave surface, we introduce a powerful and relatively simple method of analyzing control strategies for second-harmonic generation and parametric frequency conversion. The analysis described below extends the standard theory of quasi-phase matching, $, 4,7,15$ in which the three-wave equations are linearized with the undepleted-pump approximation. It complements the previous analysis of quasi-phase matching that allows pump depletion, ${ }^{5}$ by enabling a simple visual and qualitative understanding of the results without explicit analysis of elliptic integrals.

The paper is organized as follows. First we review the Hamiltonian structure of the three-wave system (Section 2), emphasizing the connection between the ManleyRowe relations and phase symmetries. These phase symmetries are then used to reduce the three-wave system to a system in three real variables $X, Y$, and $Z$, chosen to be invariant under the phase symmetries (Section 3 ). The dynamics in $(X, Y, Z)$ space is shown to be confined to a closed cubic surface of revolution, the three-wave surface. The trajectories are given by intersections of the three-wave surface with a family of parallel planes, whose slope measures the relative strength of the wave-vector mismatch and the quadratic nonlinearity (Section 4). This geometric realization of the three-wave dynamics is then used to analyze techniques for controlling frequency conversion in $\chi^{(2)}$ media, and approximations are obtained when the phase mismatch dominates. We describe quasi-phase matching of both type I and type II second-harmonic generation, as well as generic parametric three-wave interactions, using the three-wave surface (Section 5).

\section{THREE-WAVE EQUATIONS AND THEIR SYMMETRIES}

The three-wave equations, written

$$
\frac{\mathrm{d} A_{1}}{\mathrm{~d} z}=-i \gamma_{1} A_{2}^{*} A_{3} \exp (-i \Delta k z)
$$

$$
\begin{aligned}
& \frac{\mathrm{d} A_{2}}{\mathrm{~d} z}=-i \gamma_{2} A_{1}^{*} A_{3} \exp (-i \Delta k z), \\
& \frac{\mathrm{d} A_{3}}{\mathrm{~d} z}=-i \gamma_{3} A_{1} A_{2} \exp (i \Delta k z),
\end{aligned}
$$

are obtained as a slowly varying amplitude approximation of Maxwell's equations for the resonant interaction of three light waves propagating in a noncentrosymmetric material. ${ }^{4}$ Here the $A_{j}$ are the slowly varying envelopes, and each has a different carrier frequency. The choice of the $\gamma_{j}$ defines the optical interaction as well as the units or normalization of the complex amplitudes. The amplitudes are typically related to units of power or intensity both in the literature ${ }^{4}$ and in texts on nonlinear optics. ${ }^{1,2}$

Both second-harmonic generation and parametric frequency conversion are modeled by the three-wave equations, where each $A_{j}$ is a complex light-wave amplitude, $\Delta k=k_{1}+k_{2}-k_{3}$ is the wave-vector mismatch, and each $\gamma_{j}$ is proportional to the second-order susceptibility, $\chi^{(2)}$, of the process. For type II second-harmonic generation, waves one and two, having the same frequency, propagate in different polarization states and interact to generate a third light wave at the second-harmonic frequency. In the limit $A_{1}=A_{2}$ and $\gamma_{1}=\gamma_{2}$ the equations for type I second-harmonic generation are obtained. If this condition is true for the initial conditions, it continues to be the case for all time. Thus the condition is dynamically invariant, and type I second-harmonic generation is always a special case of the three-wave system.

In what follows it is convenient for us to shift into a rotating frame and to rescale the wave amplitudes by making the transformation $A_{j}=\left|\gamma_{j}\right| q_{j} \exp (-i \Delta k z)$; thus the three-wave equations become

$$
\begin{aligned}
& \frac{\mathrm{d} q_{1}}{\mathrm{~d} \xi}=i \Delta \Gamma q_{1}-i s q_{2}^{*} q_{3}, \\
& \frac{\mathrm{d} q_{2}}{\mathrm{~d} \xi}=i \Delta \Gamma q_{2}-i s q_{1}^{*} q_{3}, \\
& \frac{\mathrm{d} q_{3}}{\mathrm{~d} \xi}=i \Delta \Gamma q_{3}-i s q_{1} q_{2},
\end{aligned}
$$

where $\xi=z \sqrt{\left|\gamma_{1} \gamma_{2} \gamma_{3}\right|}$ is the scaled propagation length, and $\Delta \Gamma=\Delta k / \sqrt{\left|\gamma_{1} \gamma_{2} \gamma_{3}\right|}$ is the relative strength of the mismatch compared with the nonlinearity. Here $s$ $= \pm 1$ is the sign of the coefficient of the quadratic nonlinearity. Notice that along with the initial data, solutions of this system depend only on the parameters $\Delta \Gamma$ and $s$. The parameter $\Delta \Gamma$ plays a crucial role in determining the qualitative dynamics. The parameter $s$ models the reversal of the crystal orientation, and it will be used in the analysis of quasi-phase matching. Written in this form, the three-wave system has the canonical Hamiltonian structure

$$
\frac{\mathrm{d} q_{j}}{\mathrm{~d} z}=\left\{q_{j}, H\right\}=-2 i \frac{\partial H}{\partial q_{j}^{*}},
$$

where the Hamiltonian, 


$$
H=\frac{s}{2}\left(q_{1}^{*} q_{2}^{*} q_{3}+q_{1} q_{2} q_{3}^{*}\right)-\frac{\Delta \Gamma}{2} \sum_{j=1}^{3}\left|q_{j}\right|^{2},
$$

is cubic in the wave amplitudes. The Poisson bracket $\{$, produces

$$
\begin{aligned}
\left\{q_{i}, q_{j}\right\} & =0, \\
\left\{q_{i}, q_{j}^{*}\right\} & =-2 i \delta_{i j} .
\end{aligned}
$$

In addition to the Hamiltonian itself, the Manley-Rowe relations

$$
\begin{aligned}
& K_{1}=\frac{1}{2}\left(\left|q_{1}\right|^{2}-\left|q_{2}\right|^{2}\right), \\
& K_{2}=\frac{1}{2}\left(\left|q_{2}\right|^{2}+\left|q_{3}\right|^{2}\right), \\
& K_{3}=\frac{1}{2}\left(\left|q_{1}\right|^{2}+\left|q_{3}\right|^{2}\right),
\end{aligned}
$$

are constants of the motion. Since any two $K_{j}$ along with $H$ form a complete and independent set of conserved quantities, i.e., $\left\{K_{i}, K_{j}\right\}=0$ and $\left\{K_{i}, H\right\}=0$, the system can be solved and solutions expressed in terms of elliptic integrals. We usually carry out this procedure by transforming the complex envelope amplitudes to real coordinates $\rho_{j}, \phi_{j}$ defined by $q_{j}=\rho_{j}^{\beta} \exp \left(i \phi_{j}\right)$, where $\beta$ may be either 1 or $1 / 2$. Using the Manley-Rowe invariants (6a)(6c), we reduced the system to a one degree of freedom potential equation and then to quadratures by eliminating the dependence on each of the other variables. ${ }^{4-8}$

Analysis of this nonlinear oscillator and the associated elliptic integrals then provides information about the dynamics of the system. The phases may also be studied in terms of integrals of elliptic functions. ${ }^{17,18}$

Notice that this procedure reduces the three-wave phase space to that of a one-dimensional nonlinear oscillator. Unfortunately, only information about a single wave amplitude (and indirectly the relative phase) of the system is retained in this representation. By exploiting the fact that the Manley-Rowe invariants correspond to symmetries of the three-wave system, we reduce the three-wave phase space to a closed two-dimensional surface in three dimensions. This representation of the dynamics retains information about all of the amplitudes and the relative phase.

The phase-space coordinates are chosen to be invariant with respect to symmetries connected to the ManleyRowe invariants (6a)-(6c). These symmetries manifest themselves in the three-wave equations as invariants with respect to phase shifts. One way to understand this is to let each of the $K_{j}$ act as the "Hamiltonian" in the canonical equations of motion (3). The dynamics generated by each $K_{j}$ introduces phase shifts, a unitary transformation, on the vector of three-wave amplitudes. In real coordinates $q_{j}=x+i y$ these are just the motions of uncoupled harmonic oscillators. The specific phase shifts associated with the Manley-Rowe symmetries are

$$
\begin{aligned}
& \left(q_{1}, q_{2}, q_{3}\right) \rightarrow\left(q_{1} \exp \left(-i \theta_{1}\right), q_{2} \exp \left(i \theta_{1}\right), q_{3}\right), \\
& \left(q_{1}, q_{2}, q_{3}\right) \rightarrow\left(q_{1}, q_{2} \exp \left(-i \theta_{2}\right), q_{3} \exp \left(-i \theta_{2}\right)\right), \\
& \left(q_{1}, q_{2}, q_{3}\right) \rightarrow\left(q_{1} \exp \left(-i \theta_{3}\right), q_{2}, q_{3} \exp \left(-i \theta_{3}\right)\right),
\end{aligned}
$$

for $K_{1}$ through $K_{3}$, respectively. It is easily checked that the three-wave equations $(2 \mathrm{a})-(2 \mathrm{c})$ are invariant under these transformations.

\section{REDUCTION TO THREE-WAVE SURFACES}

If a system of first-order ordinary differential equations has a phase symmetry, we can reduce its dimension by one by ignoring the value of the phase in question. If the system is Hamiltonian, its dimension can be reduced by two; associated with the phase symmetry is a conserved quantity, and fixing its value eliminates an additional degree of freedom. The theory of reduction generalizes this procedure in a systematic way. ${ }^{9}$

Projection of polarization dynamics onto the Poincaré sphere is a simple example of reduction. The dynamics of the two polarization components are modeled by two coupled complex amplitude equations. There are typically no frequency-mixing terms, but the equations conserve the total intensity. Since the equations are Hamiltonian, intensity conservation reduces the dimension of the system from four to two. This conserved quantity is also associated with a phase symmetry of the equations. The Stokes parameters ${ }^{12}$ are invariant coordinates with respect to this phase symmetry that project the dynamics onto the Poincare sphere. The dynamics of the amplitudes and the relative phase of the polarization components are retained in the reduced phase space, but the absolute phase is not. In the following analysis, reduction is applied to three-wave interactions.

We achieve reduction of the three-wave system by introducing new coordinates that are invariant with respect to the phase symmetries (7a)-(7c) associated with the Manley-Rowe relations. As noted above, only two of these symmetries are independent. Since the three-wave system has three complex, or six real, dimensions, it follows that there are four independent phase-invariant coordinates. The coordinates $(X, Y, Z)$ defined by

$$
\begin{aligned}
X+i Y & =q_{1} q_{2} q_{3}^{*}, \\
Z & =\sum_{j=1}^{3} p_{j}\left|q_{j}\right|^{2},
\end{aligned}
$$

where the $p_{j}$ are real constants, are clearly invariant under the phase symmetries, as are the associated conserved quantities $K_{1}, K_{2}$, and $K_{3}$ of the Manley-Rowe relations. Thus we expect two relations among the invariants $X, Y, Z$ and $K_{1}, K_{2}, K_{3}$. We have already noted the relation $K_{3}=K_{1}+K_{2}$. We obtain the second relation by taking the square modulus of $X+i Y$ and expressing the $\left|q_{j}\right|^{2}$ that come from the right-hand side of Eq. (8a) in terms of $Z$ and the Manley-Rowe relations. This yields the relation $\phi=0$, where

$$
\begin{aligned}
\phi & =-\eta\left(X^{2}+Y^{2}\right)+\kappa\left(Z-Z_{1}\right)\left(Z+Z_{2}\right)\left(Z-Z_{3}\right), \\
\kappa & =1 / \eta^{2}, Z_{1}=2\left(p_{3} K_{2}+p_{1} K_{1}\right), \\
Z_{2} & =2\left(p_{1} K_{3}+p_{2} K_{2}\right), \\
Z_{3} & =-2\left(p_{2} K_{1}-p_{3} K_{3}\right),
\end{aligned}
$$


The function $\phi=0$ implicitly defines for given $K_{1}, K_{2}$, and $K_{3}$ the three-wave surface in $(X, Y, Z)$ space. Since the $K_{j}$ are also constants of the motion, the three-wave dynamics is captured by the motion dynamics of $(X, Y, Z)$. The coordinate $Z$ can be chosen to provide a convenient comparison among intensities of the three waves. For second-harmonic generation, one might choose $p=(-1,-1,1)$, so $Z$ provides a measure of the conversion efficiency. More light at the second harmonic corresponds to larger values of $Z$. By making the new coordinates invariant with respect to the Manley-Rowe phase symmetries, we have removed information about the absolute phases of each wave. The new coordinates $(X, Y, Z)$ include information only about the amplitudes and the relative phase among the waves.

Compared with the standard variable transformation $q_{j}=\rho_{j}^{\beta} \exp \left(i \phi_{j}\right)$, the coordinates $X$ and $Y$ may be regarded as the components of a vector of length $\left|\rho_{1} \rho_{2} \rho_{3}\right|^{\beta}$ and polar angle $\Omega=\left(\phi_{1}+\phi_{2}-\phi_{3}\right)$, where $\Omega$ is the relative phase of the three waves. If $p_{j}=1$ is chosen for any one value of $j$ and if $p_{j}=0$ is chosen for each of the other two values of $j$, then $Z=\rho_{j}^{2 \beta}$. This choice of $Z$ provides a direct connection to the standard analysis in the case of a depleted pump.

Using the invariant coordinates $X, Y$, and $Z$ defined in Eqs. (8a) and (8b), we can rewrite the three-wave system and its Hamiltonian structure. We can obtain the reduced equations of motion by differentiating the expressions for $(X, Y, Z)$ in Eqs. (8a) and (8b) and by removing all instances of $q_{j}$ by substitution. It is often more convenient to transform the equations written in terms of Poisson brackets (3). With the reduced coordinates, the Poisson structure of the three-wave system becomes

$$
\frac{\mathrm{d} W_{j}}{\mathrm{~d} \xi}=\left\{W_{j}, H_{r}\right\}, j=1,2,3,
$$

where $W=(X, Y, Z)$ and $H_{r}$ is the Hamiltonian (4) written by use of the reduced coordinates. The reduced equations of motion are obtained once the reduced Hamiltonian and Poisson brackets among the reduced coordinates are known. By direct substitution using Eqs. (8a) and (8b), we obtain the reduced Hamiltonian

$$
H_{r}=s X-\frac{\Delta \Gamma}{2 \eta}(Z+2 \delta),
$$

where, as above, $\eta=p_{1}+p_{2}-p_{3}$ and $\delta=\left(p_{2}-p_{1}\right)$ $\times K_{1}+\left(p_{2}-p_{3}\right) K_{2}+\left(p_{1}-p_{3}\right) K_{3}$. This choice of coordinates leads to the important simplification that the reduced Hamiltonian is linear in $X$ and $Z$ and independent of $Y$. We obtain the reduced Poisson brackets,

$$
\begin{aligned}
& \{X, Y\}=-\partial \phi / \partial Z, \\
& \{Z, X\}=-2 \eta Y, \\
& \{Y, Z\}=-2 \eta X,
\end{aligned}
$$

by computing the brackets (5a) and (5b) among the invariant coordinates $(X, Y, Z)$. Using these results, we write the reduced three-wave equations explicitly as

$$
\frac{\mathrm{d} X}{\mathrm{~d} \xi}=-\Delta \Gamma Y
$$

$$
\begin{aligned}
& \frac{\mathrm{d} Y}{\mathrm{~d} \xi}=\Delta \Gamma X+s \frac{\partial \phi}{\partial Z}, \\
& \frac{\mathrm{d} Z}{\mathrm{~d} \xi}=-2 s \eta Y .
\end{aligned}
$$

In this form it is clear that the coupling between $X$ and $Y$ is equivalent to that of a harmonic oscillator with frequency $\Delta \Gamma$, and the coupling between $Y$ and $Z$ is equivalent to a nonlinear oscillator. These equations can also be written in the compact form

$$
\frac{\mathrm{d} W}{\mathrm{~d} \xi}=\nabla_{r} H_{r} \times \nabla_{r} \phi,
$$

where the gradient is taken with respect to the reduced coordinates as $\nabla_{r}=(\partial / \partial X, \partial / \partial Y, \partial / \partial Z)$. Since $\mathrm{d} H_{r} / \mathrm{d} \xi$ $=\nabla_{r} H_{r} \cdot \mathrm{d} W / \mathrm{d} \xi$ and $\mathrm{d} \phi / \mathrm{d} \xi=\nabla_{r} \phi \cdot \mathrm{d} W / \mathrm{d} \xi$, it is clear from Eq. (14) that $H_{r}$ and $\phi$ are constants of the motion.

We integrate the reduced equations (13a)-(13c) by differentiating Eq. (13c) with respect to $\xi$ and substituting (13b). The result is a potential equation for $Z$ :

$$
\frac{1}{2}\left(\frac{\mathrm{d} Z}{\mathrm{~d} \xi}\right)^{2}+U=E
$$

where the reduced Hamiltonian, $H_{r}$, has been used to replace $X$, and the "energy" $E$ is the constant of integration. The potential, $U$, is given by

$$
U=2 \eta[\phi(X=0, Y=0, Z)+r Z]+\frac{1}{2} \Delta \Gamma^{2} Z^{2},
$$

where $r=\Delta \Gamma H_{r}+\Delta \Gamma^{2} \delta / 2 \eta$. Integrating the potential equation, we find that

$$
\xi-\xi_{0}=\int \frac{\mathrm{d} Z}{\sqrt{2[E-U(Z)]}},
$$

where $E-U(Z)$ is a cubic polynomial for which the sign of the coefficient of the highest power is given by the sign of $\eta$. Defining $\zeta_{1}>\zeta_{2}>\zeta_{3}$ to be real roots of $E$ - $U(Z)$ with $\eta>0$, then $E-U(Z) \geqslant 0$ on $\left(\zeta_{2}, \zeta_{3}\right)$ and

$$
\xi-\xi_{0}=\left(\frac{1}{2 k}\right) \int \frac{\mathrm{d} \theta}{\sqrt{1-M \sin ^{2}(\theta)}}
$$

where $M=\left(\zeta_{2}-\zeta_{3}\right) /\left(\zeta_{1}-\zeta_{3}\right)$ and $k=\left[\left(\zeta_{1}-\zeta_{3}\right) / 2\right]^{1 / 2} / 2$. In this case the solution is

$$
Z(\xi)=\zeta_{2}-\left(\zeta_{2}-\zeta_{3}\right) \operatorname{cn}^{2}\left\{\left[\left(\zeta_{1}-\zeta_{3}\right) / 2\right]^{1 / 2}\left(\xi-\xi_{0}\right) \mid M\right\},
$$

where $\zeta_{3}$ is the minimum value of $Z$ and $\zeta_{2}$ is the maximum value of $Z$ over a period. The positions of the roots $\zeta_{j}$ are specified by the values of the initial data and the parameters $s$ and $\Delta \Gamma$. We obtain this dependence by comparing the coefficients of the polynomial $E-U(Z)$ with those of its factored form. When one of the roots $\zeta_{j}$ can be written in terms of the extremal values of $Z$ (and therefore the $q_{j}$ ) the other two are then obtained algebraically. 5,8

The period of energy transfer among the waves is given as 


$$
L=\oint \frac{\mathrm{d} Z}{\sqrt{2[E-U(Z)]}}=\frac{K(M)}{k},
$$

where the loop integral is taken about neighboring roots over which $E-U(Z) \geqslant 0$ and $K(M)$ is the complete elliptic integral of the first kind. If $Z$ is defined so that only one $p_{j}$ is nonzero, the one-dimensional potential equation obtained by the standard method with variables $\rho_{j}^{\beta} \exp (i \phi)$ is recovered. Once $Z$ is known, the value of $X$ is obtained with the reduced Hamiltonian, $H_{r}$, and the value of $Y$ is then obtained with $\phi=0$. To reconstruct the solution of the three-wave equations, the wave amplitudes are then computed in terms of $Z$ and their initial values through Manley-Rowe invariants $\left(K_{1}, K_{2}, K_{3}\right)$. The relative phase is $\arg (X+i Y)$. The two remaining phases are not given in terms of algebraic relations. They must be integrated. We can do this by using the variables $q_{j}=\rho_{j}^{\beta} \exp \left(i \phi_{j}\right)$ and by integrating $\mathrm{d} \phi_{j} / \mathrm{d} \xi \cdot{ }^{17,18}$ Recently the geometric structure of the three-wave system was exploited to obtain these phase shifts. ${ }^{10}$ In general these shifts have a geometric and a dynamic component. The geometric component is proportional to the symplectic area enclosed by an orbit on the three-wave surface.

\section{REDUCED PHASE SPACE}

The three-wave system has now been reduced to a set of equations in three space. These reduced equations produce motion confined to a closed surface in $(X, Y, Z)$. In this section we describe how to generate the reduced phase space by constructing orbits on the three-wave surfaces. We then show how to interpret the dynamics of wave mixing in terms of motion on the reduced phase space. Recall that on these three-wave surfaces, only the dynamics of the relative phase and the amplitudes of the waves are captured. In many applications the absolute phases are not needed, but they can be obtained analytically as described above.

A. Geometric Construction of the Reduced Phase Space First, recall that Eq. (14) implies $H_{r}$ and $\phi$ are constants of the motion. Since $H_{r}$ is linear, its level sets are the planes

$$
Z=m X-\left(s m H_{r}+2 \delta\right),
$$

with slopes $m=2 s \eta / \Delta \Gamma$. Expressed in terms of the wave amplitudes $q_{j}, \phi(X, Y, Z)$ vanishes identically. Thus the reduced dynamics is confined to the surfaces given by $\phi(X, Y, Z)=0$. These three-wave surfaces are generically egg-shaped. Notice that in the case of polarization dynamics, $\phi(X, Y, Z)$ is quadratic in $Z$ and the analogous surfaces are spheres.

Trajectories of the reduced equations are the curves produced by intersecting the level sets of $H_{r}$ with the three-wave surfaces. Figure 1 shows the intersection of a three-wave surface and a planar level set of $H_{r}$. This intersection defines a trajectory of the system.

A typical reduced phase space on a three-wave surface is plotted in Fig. 2 and corresponds to either type II second-harmonic generation or parametric three-wave in- teractions. Apart from fixed points, the three-wave surface is filled with periodic orbits. As the system traverses one of these cycles, light is converted from the pump wave to the signal and idler waves and then back to the pump wave.

Type I second-harmonic generation is realized when $K_{2}=K_{3}$. In this limit a pair of the roots of $\phi(X=0$, $Y=0, Z)=0$ coalesce, and a singular point forms. A typical phase space for type I second-harmonic generation is plotted in Fig. 3.

\section{B. Features of the Reduced Phase Space}

We define the shape and size of the three-wave surface by fixing the values of the Manley-Rowe invariants, which are defined by the initial values of the scaled wave amplitudes $\left(\left|q_{1}\right|,\left|q_{2}\right|,\left|q_{3}\right|\right)$. The plane defined by the corresponding Hamiltonian must pass through the initial point in $(X, Y, Z)$ as defined by the initial amplitudes and phases of $\left(q_{1}, q_{2}, q_{3}\right)$. The value of $m$ then fixes the orbit associated with this initial point. After one trip around the three-wave surface, the reduced system returns to its initial position. The amplitudes and the relative phase return to their initial values.

The qualitative features of the phase space depend only on the slope $m$ and the invariants $K_{j}$. These parameters

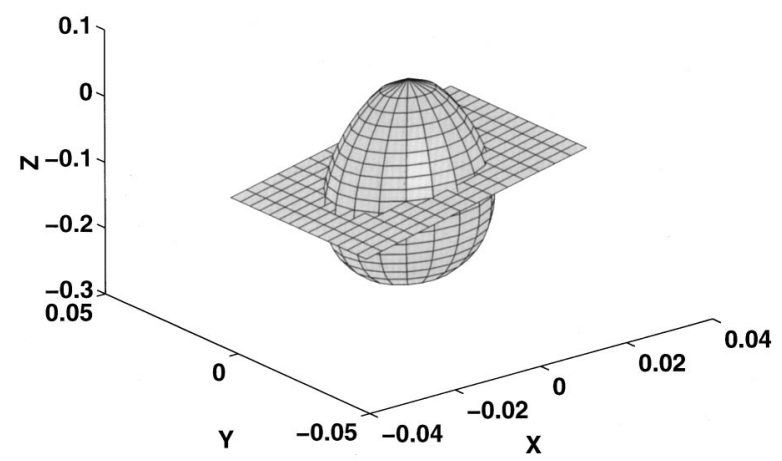

Fig. 1. Orbit of the reduced three-wave equations is constructed by intersection of a three-wave surface with a plane that is one level set of the reduced Hamiltonian. Here $p=(-1,-1,1), s$ $=1, \Delta \Gamma=40$, and the Manley-Rowe relations are defined by $\left(q_{1}(0), q_{2}(0), q_{3}(0)\right)=(0.1,0.6,1.0)$.

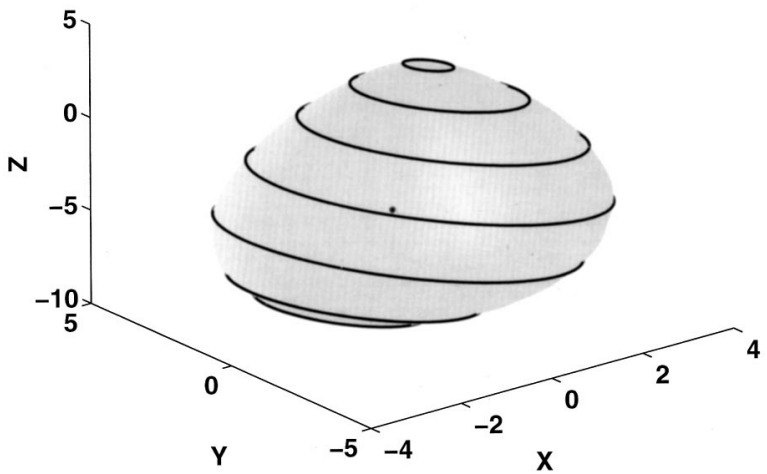

Fig. 2. Reduced three-wave phase space for type II secondharmonic generation or parametric frequency conversion on a three-wave surface. Here, $p=(-1,-1,1), s=1, \Delta \Gamma=10$, and the Manley-Rowe relations are defined by $\left(q_{1}(0), q_{2}(0), q_{3}(0)\right)=(1.0,0.5,2.0)$. 


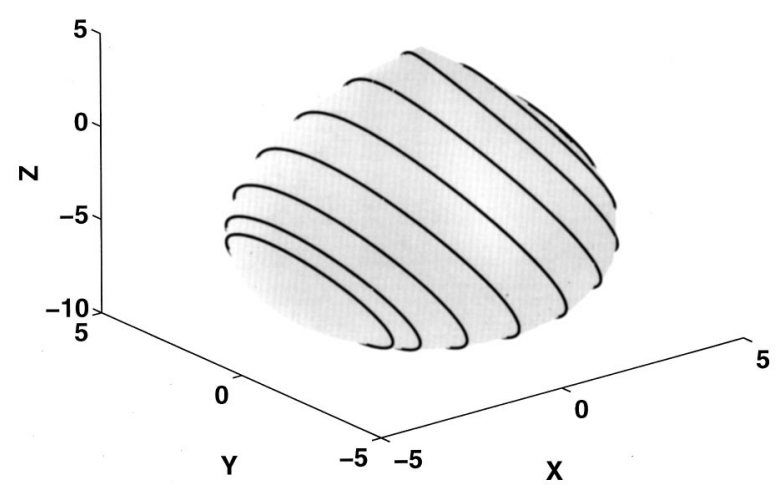

Fig. 3. Reduced three-wave phase space for type I secondharmonic generation on a three-wave surface, where $p$ $=(-1,-1,1), s=1$, and $\Delta \Gamma=2$. The Manley-Rowe relations are defined by $\left(q_{1}(0), q_{2}(0), q_{3}(0)\right)=(1.0,1.0,2.0)$, so $K_{2}=K_{3}$.

define the interactions of the three light waves. For a fixed quadratic coefficient, $m$ varies inversely with the phase mismatch. Near phase matching, $\Delta k \ll 1$, so $\Delta \Gamma$ $\ll 1$ and the slope $m$ is large, meaning the Hamiltonian planes tend to be vertical. Far from phase matching the slope $m$ is small and the Hamiltonian planes are nearly horizontal.

The three-wave surface is always a single closed surface. For a given choice of the $p_{j}$, the $K_{j}$ define the positions of the roots of $\phi(X=0, Y=0, Z)=0$. One root coincides with the top and a neighboring root coincides with the bottom of the three-wave surface. A consistent set of initial data must lie between these two roots, and all points on the trajectory that it generates must lie between the same pair of roots. This is necessary in order that $X, Y$, and $Z$ remain real.

An important feature that simplifies the analysis of controls is that the three-wave surfaces are independent of the value of $\Delta \Gamma$ and $s$, so the transformation $s \rightarrow-s$, which corresponds to changing the sign of the secondorder susceptibility, $\chi^{(2)}$, leaves the three-wave surface invariant.

In these calculations the vector $p$ provides some flexibility in the definition of $Z$ as a linear combination of the squares of the field amplitudes. In this way, $Z$ can be chosen to be proportional to the amount of conversion. For a given $\chi^{(2)}$ process the conversion efficiency can then be extracted directly from the phase portrait on the threewave surface. If $p$ is chosen so that the orbit passing through the highest point on the surface produces complete conversion, then for a given initial condition, $m$ can be chosen so that the trajectory passes through the point at the top of the three-wave surface. The process of varying $m$ is analogous to tuning the phase mismatch, $\Delta k$. Consider the choice $p=(-1,-1,1)$. When $\Delta k=0$, the orbit passing through $(X, Y)=(0,0)$ produces maximum conversion.

Relative fixed points, sometimes called eigenmodes, are located at the points where the Hamiltonian planes are tangent to the three-wave surface. The value of $Z$ remains constant at these points, so no energy is exchanged. These points are generically elliptic points. For $K_{2}=K_{3}$, as in the case of type I second-harmonic generation, an additional relative fixed point appears at the singular point. The trajectory connected to it is homoclinic since it connects a fixed point to itself. The homoclinic trajectory, given by $H_{r}=0$, divides the threewave surface in half, with $H_{r}>0$ on one side and $H_{r}$ $<0$ on the other. This orbit corresponds to the wellknown tanh solution. In the phase-matched system, where $m \gg 1$, the pair of elliptic points are located on the sides of the three-wave surfaces. As $m$ decreases and the mismatch increases, one of these points migrates toward the top and one toward the bottom. If $K_{2}=K_{3}$, the homoclinic orbit shrinks as one of the elliptic points approaches the homoclinic point, and the portion of the phase space having characteristically nonlinear features shrinks. In the limit $m=0$ the area enclosed by the homoclinic is zero and the motion is purely linear.

\section{Linearization and the Undepleted-Pump Approximation}

In many applications we linearize the three-wave system by assuming that the pump wave remains nearly constant throughout the evolution of the wave interaction. This is always the situation near the elliptic fixed points since only small variations in the $Z$ coordinate occur there. As the phase mismatch becomes large compared with the strength of the nonlinear interaction, so that $\Delta \Gamma \gg 1$, the slope of the level sets of the reduced Hamiltonian $H_{r}$ become small ( $m \ll 1$ ). In this limit the $Z$ coordinate makes small, nearly sinusoidal oscillations near its initial value. This corresponds to the undepletedpump limit.

For interactions over a single period the reduced threewave system (13a)-(13c) can be linearized in the limit $\Delta \Gamma \gg 1$. Take $\Delta \Gamma=\Gamma / \epsilon$, where $0<\epsilon \ll 1$. We rescale the evolution variable so $T=\xi / \epsilon$ and obtain

$$
\begin{aligned}
& \frac{\mathrm{d} X}{\mathrm{~d} T}=-\Gamma Y, \\
& \frac{\mathrm{d} Y}{\mathrm{~d} T}=\Gamma X+s \epsilon \frac{\partial \phi}{\partial Z}, \\
& \frac{\mathrm{d} Z}{\mathrm{~d} T}=-2 s \epsilon \eta Y .
\end{aligned}
$$

Now we linearize by letting $W=(X, Y, Z)=W_{0}$ $+\epsilon \delta W$. To leading order in $\epsilon$,

$$
\begin{aligned}
& \frac{\mathrm{d} X_{0}}{\mathrm{~d} T}=-\Gamma Y_{0}, \\
& \frac{\mathrm{d} Y_{0}}{\mathrm{~d} T}=\Gamma X_{0}, \\
& \frac{\mathrm{d} Z_{0}}{\mathrm{~d} T}=0 .
\end{aligned}
$$

Consistent with the undepleted-pump approximation, no conversion takes place. At this order the system simply oscillates in the $(X, Y)$ plane with frequency $\Gamma$. This corresponds to a period of $2 l_{c}=2 \pi / \Delta \Gamma$. In the physical coordinates, $l_{c}$ is $\pi / \Delta k$. Note that when $K_{2}=K_{3}$, special 
care must be taken near the homoclinic orbit, but as noted above the area enclosed by this orbit tends to zero as $m$ tends to zero.

At the next order in $\epsilon$, the dynamics is governed by the equations

$$
\begin{aligned}
& \frac{\mathrm{d} \delta X}{\mathrm{~d} T}=-\Gamma \delta Y, \\
& \frac{\mathrm{d} \delta Y}{\mathrm{~d} T}=+\Gamma \delta X+\left.s \frac{\partial \phi}{\partial Z}\right|_{Z=Z_{0}}, \\
& \frac{\mathrm{d} \delta Z}{\mathrm{~d} T}=-2 s \eta \delta Y .
\end{aligned}
$$

These equations reveal that the first correction to the oscillation in the $(X, Y)$ plane is to shift its center along the $X$ direction by introducing a constant driving term determined by the value of $Z_{0}$. In addition, there is now motion in the $Z$ coordinate. This motion is slaved to the rate of change of the $Y$ coordinate and is thus a small oscillation. As expected, this gives a small amount of conversion and introduces motion perpendicular to the $(X, Y)$ plane.

For $m \ll 1$ the linear approximation is valid for at least propagation distances of the order of a period for most orbits in the reduced phase space, so for large phase mismatch the linear approximation will be sufficient for distances of order $\pi / \Delta k$. Notice that secular terms enter at the next order in this expansion since the amplitude dependence of the period is ignored. This amplitude dependence or Stokes shift of the period is obtained with a multiple time scales or averaging approach.

The leading-order oscillation in $Z$ can also be obtained from the explicit solution for $Z$ in Eq. (19). Let $a=\left(\zeta_{1}\right.$ - $\left.\zeta_{2}\right) / 2$. Then $M=2 a /\left(\zeta_{2}-\zeta_{3}\right)$, and, as the amplitude of the oscillation in $Z$ tends to zero, $a \rightarrow 0, M \rightarrow 0$, and $Z \rightarrow \zeta_{2}-a\left\{1-\cos \left[k\left(\xi-\xi_{0}\right)\right]\right\}+O\left(a^{2}\right)$, where $k=\left[\left(\zeta_{1}\right.\right.$ $\left.\left.-\zeta_{3}\right) / 2\right]^{1 / 2}$. In this limit the period is $2 \pi / k$. We calculate the correction of the period that is due to nonlinear terms as

$$
L=\frac{\pi}{k}\left[1+\left(\frac{1}{2}\right)^{1 / 2} M+\ldots\right]
$$

by expanding the expression (20) for $L$ in the small amplitude limit.

\section{CONTROL VISUALIZED ON THREE- WAVE SURFACES}

In this section we analyze the control of energy flow among the three light waves. In previous work ${ }^{5}$ a potential equation analogous to Eq. (15) was solved directly in $(\rho, \phi)$ coordinates, and quasi-phase matching was analyzed with the solutions. We can visualize these solutions by considering either deformations of the potential $U(Z)$ or the phase portrait in $(Z, \mathrm{~d} Z / \mathrm{d} \xi)$ as the values of the parameters $s$ and $\Delta \Gamma$ are varied. Here we use the phase space on the three-wave surface to visualize the transfer of energy among the three light waves. In this setting the phase portrait deforms in a simple way as the parameters $s$ and $\Delta \Gamma$ vary, and it is easy to see how to manipulate the dynamics of the three-wave interaction. On the reduced three-wave surface, information about all three wave amplitudes as well as the relative phase is retained. As noted above, only information about the absolute phases was removed during the process of symmetry reduction.

Controlling the transfer of energy among the light waves can be analyzed as a motion planning problem on a three-wave surface. In the three-wave equations (2a)(2c) either the linear or the nonlinear term may play the role of the control vector field. The goal is to move from a point on the three-wave surface associated with the initial state of the three-wave system to a second point associated with maximum conversion. As noted earlier, one way to do this is to vary the phase-matching parameter until the initial point is connected to the final point with a single orbit. In practice this is achieved by temperature or angle tuning of the crystal to phase match the waves. This procedure is often difficult to implement in devices and reduces their robustness.

When the system is not perfectly phase matched, an initial point on the three-wave surface can still be connected to any final point by modulation of the material parameters. For instance, if the phase-space velocities for $s=1$ and $s=-1$ are independent at each point of the three-wave surface, then the reduced system is controllable; any pair of points can be joined by a finite concatenation of $s=1$ and $s=-1$ flows. As the parameters are modulated, a point on the three-wave surface is first pushed in one direction and then in another. In this way any point on the three-wave surface can be reached in finite time and the three-wave system can be controlled up to the value of the absolute phases.

When the reduced phase portraits associated with the two different choices of material parameters share a fixed point, the system is no longer controllable. In the threewave system, such an exceptional point appears when $K_{2}=K_{3}$. This is also the case of type I second-harmonic generation. Here both vector fields approach the fixed point along a homoclinic orbit. Even in this special but important case it is possible to get as close in finite time as needed in optical devices. What is more, for large phase mismatch the region of the phase space that is affected is small. Thus the basic requirement to achieve control is that the modulated parameter or parameters produce at least two different directions for the phasespace velocity vectors at nearly all points on the threewave surface.

These basic ideas allow a complete understanding of the control of frequency conversion in the fully nonlinear regime and are based on a very simple geometric construction that permits all the basic features to be visualized. We consider two specific cases below.

\section{A. Quasi-Phase Matching}

Quasi-phase matching is a robust and efficient way to enhance the effective conversion efficiency of optical materials, and it has a simple geometric interpretation. We achieved quasi-phase matching by alternating the sign of $\chi^{(2)}$ at every half-period of the oscillation cycle (i.e., the coherence length $l_{c}=\pi / \Delta k$ ), which for small $m$ was seen 
above to be half the linear oscillation period or nearly half a trip around the three-wave surface. Just as conversion from the pump saturates and begins to reverse, the sign of $\chi^{(2)}$ is inverted. This reverses the direction of the conversion process so that more conversion from the pump can take place.

To understand quasi-phase matching geometrically, notice that the transformation $\gamma_{j} \rightarrow-\gamma_{j}$ or $s \rightarrow-s$ leaves the three-wave surfaces invariant but reverses the sign of $m$ and therefore the slope of the Hamiltonian planes. This leads to the following geometrical construction for quasi-phase-matching trajectories: we obtain them by concatenating intersections of the three-wave surface with Hamiltonian planes of alternating slope. In the optimal case the sign changes along $Y=0$, at a point of maximal $Z$ on one segment and minimal $Z$ on the next.

In Fig. 4 a quasi-phase-matched trajectory corresponding to type II second-harmonic generation or parametric conversion is plotted on a three-wave surface. We generated the trajectory numerically from Eqs. (2a)-(2c) by alternating the sign of $s$ after steps of size $l_{c}$. It spirals down the three-wave surface as more light is converted to the second harmonic. A similar picture is obtained in the case of type I second-harmonic generation and is plotted in Fig. 5. Here the second harmonic grows as the trajectory spirals down the three-wave surface.

To produce maximum conversion, we choose the initial data in the plane $Y=0$, where $\Omega=n \pi$ with $n$ $=0,2,4, \ldots$ if $m>0$ and $n=1,3,5, \ldots$ if $m<0$. At these points $Z$ has its minimum value and makes the maximum excursion in $Z$ on a given orbit over a halfperiod. The composite quasi-phase-matching trajectory is constructed as before; we change the sign of the quadratic coefficients each time the plane $Y=0$ is crossed. In a system where the second harmonic starts from noise, waves initially near the optimum relative phase grow most efficiently. In systems where the signal is seeded, the relative phase is tuned to achieve optimum conversion. ${ }^{19}$ If $m$ is small, the optimum conversion efficiency is closely approximated by taking steps of length $l_{c}$. Even for moderately large values of the slope $m$, conversion is improved because the period depends weakly on the value of $\Delta \Gamma$.

Looking back at Eqs. (24a)-(24c), we see that, for $m$ $\ll 1$, modulating $s$ introduces the dynamics of a driven harmonic oscillator. Thus periodically poling the quadratic coefficient introduces a periodic driving force. If its period is half the period of the oscillation, parametric instability occurs. This process eventually saturates. One way that this occurs is through the Stokes shift. In Fig. 4, dots are plotted at the points where the poling takes place. Notice that these points do not all lie along $Y=0$. The error is due to the Stokes shift. The value of this shift is given approximately in Eq. (25).

This saturation process is particularly clear as $m$ increases and the linear period becomes an increasingly poor approximation to the actual oscillation period. In this limit, quasi-phase-matching conversion saturates after only a few steps of length $l_{c}$. At these larger values of $m$ the signs of the quadratic coefficients must be alternated at half the nonlinear period to obtain the most efficient quasi-phase-matching conversion. Because this pe-

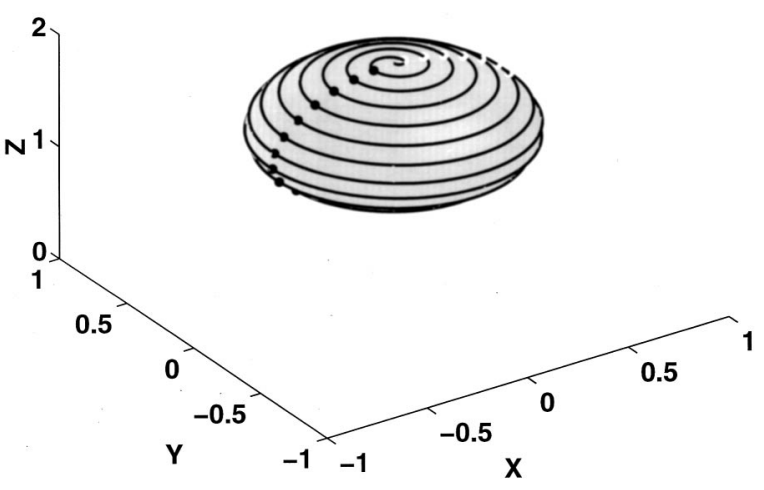

Fig. 4. Composite trajectory with 30 segments of length $l_{c}$ $=\pi / \Delta k$ for type II quasi-phase matching or parametric frequency conversion is plotted on a three-wave surface. Here $p$ $=(-1,1,1), \quad \Delta \Gamma=40, \quad$ and initially $s=1$ and $\left(q_{1}(0), q_{2}(0), q_{3}(0)\right)=(0.0,1.0,1.0)$.

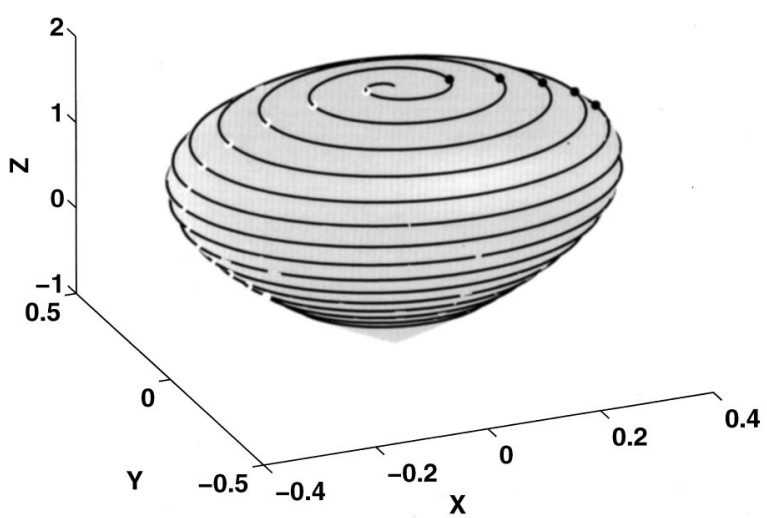

Fig. 5. Composite trajectory with 30 segments of length $l_{c}$ $=\pi / \Delta k$ for type I quasi-phase-matching second-harmonic generation. Here $p=(1,1,-1), \Delta \Gamma=40$, and initially $s=1$, and $\left(q_{1}(0), q_{2}(0), q_{3}(0)\right)=(1.0,1.0,0.0)$.

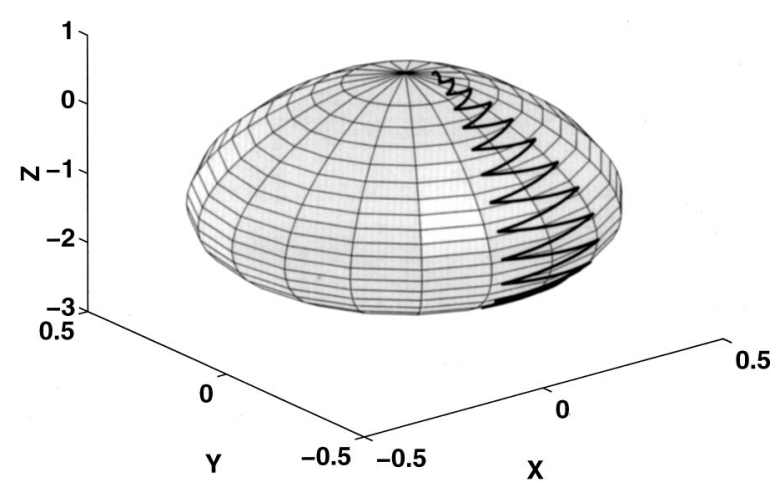

Fig. 6. Composite trajectory for the zig-zag strategy for type II second-harmonic generation. The phase mismatch is modulated after $\quad l_{c}=\pi /(2 \Delta k) \quad$ and $\quad\left(q_{1}(0), q_{2}(0), q_{3}(0)\right)=(0.1,0.6$, $\exp (i * \pi / 5))$, so the initial relative phase is near $\pi / 5$. Here $s$ $=1, \Delta \Gamma=10$, and the $Z$ coordinate is defined by $p$ $=(-1,-1,1)$.

riod varies as the harmonic grows, optimizing the conversion efficiency requires that the length of the poled segments be varied along the propagation path. If $m$ is 
large enough, only a few layers are needed to produce complete conversion. If the length of the poled segments cannot be varied, corrections to both $l_{c}$ and the initial relative phase give the constrained optimum conversion efficiency for the system. ${ }^{5}$

\section{B. Zig-Zag Control}

The usual strategy for quasi-phase matching described above is only one of many possibilities for controlling energy flow in resonant-wave interactions. Any two points on the three-wave surface can be connected by a composite trajectory if the system parameters are modulated between at least two states. In standard quasi-phase matching, the two states are the two signs of $\chi^{(2)}$. We can use an alternate strategy for the robust control of frequency conversion at any value of $m$ by modulating the sign of the mismatch parameter at a period shorter than the oscillation period for frequency conversion. The portions of the trajectories that are most nearly vertical produce the most conversion and are located near $X$ $=0$. Therefore, in contrast to the standard quasi-phasematching strategy, the optimum initial data have relative phase near $\Omega=n \pi / 2, n=1,2,3, \ldots$. Geometrically, the composite trajectory looks like a zig-zag stepping down the side of the three-wave surface along $X=0$. A composite zig-zag trajectory is plotted on a three-wave surface in Fig. 6. It moves down the three-wave surface as more light is converted. We refer to this strategy as the zigzag control.

\section{CONCLUSION}

A geometric description of frequency conversion in materials with quadratic nonlinearity has been introduced that enables the dynamics of the amplitudes and the relative phase of the waves to be viewed on a single closed surface in three dimensions. Composite trajectories constructed in the spirit of quasi-phase matching connect any two points on a three-wave surface as long as the tangent vectors of at least two families of trajectories are not parallel for most points on the reduced surface. With this approach, controls for the three-wave dynamics were analyzed, and both the zig-zag and the well-known quasiphase-matching strategies were described geometrically.

While a relatively small amount of conversion is obtained between each modulation of the parameters, the net conversion can be quite large, so the average trajectory is approximately given by a system with a much smaller value of the ratio of mismatch to nonlinearity. In this way, controlling the dynamics of the three-wave interaction enhances the net nonlinear response of the material.

Randomly distributed errors in the poling period degrade the conversion efficiency ${ }^{5,15}$ but do not introduce catastrophic errors. This robustness to errors in the poling period is easy to understand with the geometric description of quasi-phase matching on the three-wave surface. The structure of Eqs. (13a)-(13c) also illustrates important features of frequency conversion that make quasi-phase matching particularly robust.

The geometric approach for analyzing wave interactions and their control described here provides a general approach that can be used for many other systems. It underscores the idea that engineering the dynamics of wave interactions improves the net performance of optical materials.

\section{ACKNOWLEDGMENTS}

M. S. Alber was partially supported by National Science Foundation (NSF) grants DMS 9626672 and 9508711. G. G. Luther gratefully acknowledges support from The Basic Research Institute in the Mathematical Sciences, Hewlett-Packard Labs, and from NSF under grants DMS 9626672 and 9508711 . J. E. Marsden was partially supported by the California Institute of Technology and NSF grant DMS-9802106. J. M. Robbins was partially supported by NSF grant DMS 9508711, by North Atlantic Treaty Organization grant CRG 950897, and by the Department of Mathematics and the Center for Applied Mathematics, University of Notre Dame.

\section{REFERENCES}

1. A. Yariv, Quantum Electronics (Wiley, New York, 1980).

2. R. Boyd, Nonlinear Optics (Wiley, New York, 1988).

3. A. C. Newell and J. V. Moloney, Nonlinear Optics (AddisonWesley, Palo Alto, 1992).

4. J. A. Armstrong, N. Bloembergen, J. Ducuing, and P. S. Pershan, "Interactions between light waves in a nonlinear dielectric," Phys. Rev. 127, 1918-1939 (1962).

5. K. C. Rustagi, S. C. Mehendale, and S. Menakshi, "Optical frequency conversion in quasi-phase-matched stacks of nonlinear crystals," IEEE J. Quantum Electron. QE-18, 1029 (1982).

6. C. J. McKinstrie and G. G. Luther, "Solitary-wave solutions of the generalised three-wave and four-wave equations," Phys. Rev. A 127, 14 (1988)

7. S. Trillo, S. Wabnitz, R. Chisari, and G. Cappellini, "Twowave mixing in a quadratic nonlinear medium: bifurcations, spatial instabilities, and chaos," Opt. Lett. 17, 637639 (1992)

8. C. J. McKinstrie and X. D. Cao, "Nonlinear detuning of three-wave interactions," J. Opt. Soc. Am. B 10, 898-912 (1993)

9. J. Marsden and T. Ratiu, Introduction to Mechanics and Symmetry, Vol. 17 of Texts in Applied Mathematics, 2nd ed. (Springer-Verlag, New York, 1999).

10. M. S. Alber, G. G. Luther, J. E. Marsden, and J. M. Robbins, "Geometric phases, reduction and Lie-Poisson structure for the resonant three-wave interaction," Physica D 123, 271-290 (1998).

11. M. S. Alber, G. G. Luther, J. E. Marsden, and J. M. Robbins, in Proceedings of the Fields Institute Conference in Honour of the 60th Birthday of Vladimir I. Arnol'd, Fields Institute Communications Series, E. Bierstone, B. Khesin, A. Khovanskii, and J. Marsden, eds. (Field Institute, Toronto, Ontario, Canada, 1999).

12. M. Born and E. Wolf, Principles of Optics (Pergamon, Oxford, 1980).

13. D. David, D. D. Holm, and M. V. Tratnik, "Integrable and chaotic polarization dynamics in nonlinear optical beams," Phys. Lett. A 137, 355-369 (1989).

14. N. N. Akhmediev and A. Ankiewicz, Solitons (Chapman \& Hall, London, 1997)

15. M. M. Fejer, G. A. Magel, D. H. Jundt, and R. L. Byer "Quasi-phase-matched second harmonic generation: tuning and tolerances," IEEE J. Quantum Electron. 28, 26312654 (1992).

16. L. E. Myers, R. C. Eckardt, M. M. Fejer, R. L. Byer, W. R. 
Bosenberg, and J. W. Pierce, "Quasi-phase-matched optical parametric oscillators in bulk periodically poled $\mathrm{LiNbO}_{3}$," J. Opt. Soc. Am. B 12, 2102-2116 (1995).

17. A. Kobyakov, U. Peschel, and F. Lederer, "Vectorial type-II interaction in cascaded quadratic nonlinearities-an analytical approach,” Opt. Commun. 124, 184-194 (1996).
18. A. Kobyakov and F. Lederer, "Cascading of quadratic nonlinearities: a comprehensive analytical study," Phys. Rev. A 54, 3455-3471 (1996).

19. C. J. McKinstrie, G. G. Luther, and S. H. Batha, "Signal enhancement in collinear four-wave mixing," J. Opt. Soc. Am. B 7, 340-344 (1990). 